\title{
DETERMINANTES Y CONSECUENCIAS DEL TRABAJO INFANTIL: UN ANÁLISIS DE LA LITERATURA*
}

\author{
KARINA ACEVEDO GONZÁLEZ**, RAÚL QUEJADA PÉREZ*** \\ \& MARTHA YÁNEZ CONTRERAS**** \\ UNIVERSIDAD DE CARTAGENA
}

Recibido/Received/Recebido: 01/02/2011 - Aceptado/ Accepted/Aprovado: 30/04/2011

\begin{abstract}
Resumen
El presente artículo presenta los resultados de una revisión sistemática de la literatura que analiza uno de los fenómenos más complejos del mercado laboral: el trabajo infantil. El ejercicio muestra que los mayores desarrollos teóricos y empíricos se concentran en el estudio de la relación entre el trabajo de los menores y las variables de capital humano, en especial, la educación y la salud; relaciones complejas debido a que no existe una contundente evidencia de los efectos negativos del trabajo de los niños sobre estas variables. El documento destaca que con los desarrollos más recientes de la literatura se han desvirtuado hipótesis como el "luxury axiom", que atribuye el problema a la pobreza, y aparecen enfoques más amplios que incluyen los efectos de los mercados imperfectos, las migraciones y los programas de transferencias sobre el fenómeno de estudio.
\end{abstract}

Palabras clave: Producción de los hogares, trabajo infantil, educación, salud, pobreza, mercados imperfectos, migraciones.

\section{DETERMINANTS AND CONSEQUENCES OF CHILD LABOR: ANALYSIS OF LITERATURE}

\begin{abstract}
This paper presents the results of a systematic review of literature that analyses one of more complex phenomena in labor market: child labor. This exercise shows that major theoretical and empirical developments are focused in the study of minor work and human capital variables relationship, specially, education and health; complex relationships because there is not a strong evidence of the negative effects of child labor on these variables. This paper outlines that with earliest literature developments, hypothesis such as "luxury axiom" have been disorted, which attributes the problem to poverty, and appear wider approaches that include imperfect market effects, migrations and transfer programs on study phenomenon.
\end{abstract}

Keywords: Household production, child labor, education, health, poverty, imperfect markets, migrations.

Este artículo es resultado del proyecto de investigación "Trabajo infantil en la ciudad de Cartagena: un análisis transversal de sus determinantes y consecuencias en la productividad laboral futura", realizada por el grupo de Mercado Laboral de la Universidad de Cartagena (categoría D de Colciencias) como parte del convenio entre esta universidad y Colciencias para el desarrollo del programa de Joven Investigador 2010.

* Economista, Universidad de Cartagena. Joven Investigadora del Grupo de Investigación de Mercado Laboral de la Facultad de Ciencias Económicas de la Universidad de Cartagena. Correo electrónico: kacevedog@unicartagena.edu.co.

*** Economista, Universidad de Cartagena; Magister en Economía, Universidad Nacional de Colombia. Docente titular del programa de de Economía, Facultad de Ciencias Económicas de la Universidad de Cartagena. Director del Grupo de Investigación en Mercado Laboral. Correo electrónico: rquejadap@unicartagena.edu.co

Economista, Universidad de Cartagena; Magister en Economía y Magister en Economía del Medio Ambiente y de los Recursos Naturales, Universidad de los Andes. Docente titular del programa de de Economía, Facultad de Ciencias Económicas de la Universidad de Cartagena. Miembro del Grupo de Investigación en Mercado Laboral. Correo electrónico: myanezc@unicartagena.edu.co 


\title{
DETERMINANTES E CONSEQUÊNCIAS DO TRABALHO INFANTIL: UMA ANÁLISE DA LITERATURA
}

\begin{abstract}
Resumo
Este artigo apresenta os resultados de uma revisão sistemática da literatura que explora um dos mais complexos fenômenos do mercado de trabalho: o trabalho infantil. O exercício mostra que o maior avanço teórico e empírico orienta-se a estudar a relação entre trabalho infantil e as variáveis de capital humano, sobretudo educação e saúde. Essas relações são complexas, porque não há provas convincentes dos efeitos negativos do trabalho infantil sobre essas variáveis. O documento salienta que os avanços mais recentes na literatura têm refutado a hipóteses como "axioma de luxo", que atribui o problema à pobreza e a aparição de abordagens mais abrangentes que incluem os efeitos dos mercados imperfeitos, a migração e os programas de transferências sobre o fenômeno de estudo.
\end{abstract}

Palavras chave: Produção dos lares, trabalho infantil, educação, saúde, pobreza, mercados imperfeitos, migrações.

Acevedo, K., Quejada, R. \& Yánez, M. (2011) Determinantes y consecuencias del trabajo infantil: un análisis de la literatura. En: Revista de la Facultad de Ciencias Económicas de la Universidad Militar Nueva Granada. rev.fac.cienc.econ, XIX (1).

JEL: J49, I19, I21, D1.

\section{Introducción}

Las consecuencias que el trabajo infantil ejerce sobre el bienestar de los niños han sido ampliamente documentadas principalmente al interior de la literatura de corte social. Este problema ha sido abordado como un fenómeno complejo, dado que sus implicaciones trascienden en la escolaridad, la salud y la pobreza de los hogares. La vinculación temprana del niño o el joven al mercado laboral, no sólo atañe sacrificios en términos de su bienestar actual sino que además involucra un detrimento del bienestar esperado en el futuro. Todo ello en la medida que el trabajo disminuye las reservas de capital humano de los menores: en primer lugar, porque dificulta $-y$ en ocasiones obstaculiza- el tiempo dedicado a la educación con estándares de calidad aceptables; y por otro lado, porque incrementa el riesgo asociado a la exposición prolongada a ambientes de trabajo inadecuados, que implican un esfuerzo excesivo y contraproducente para el desarrollo físico y mental de los menores.
Sin embargo, en la literatura se encuentran otros enfoques que sugieren que el trabajo infantil motiva la construcción de valores que les serán de gran utilidad a los niños en la adultez para su desenvolvimiento laboral. Si bien estos enfoques reconocen que algunas formas de trabajo infantil son producto de las distorsiones económicas existentes en un país en vías de desarrollo, sus tratadistas plantean que la adquisición de habilidades, aptitudes y capacidades a temprana edad compensan las pérdidas estimadas por la no formación del capital humano. Hay que resaltar que con el trabajo infantil, se crean perfiles ocupacionales netamente empíricos, basados en la formación autodidacta, la intuición y la aplicación imperfecta de ciertas destrezas específicas, que en su conjunto terminan por perpetuar la vinculación a ocupaciones de nivel inferior y a disminuir la productividad de la labor a desarrollar en un futuro.

Todas estas características hacen del trabajo infantil un problema multidimensional y multicausal, que tiene repercusiones tanto en el corto como en el largo plazo. Es por esto que el objetivo del presente 
artículo es documentar el cuerpo teórico y empírico sobre el tema, efectuando una búsqueda sistemática en bases de datos electrónicas indexadas tales como Jstor, ScienceDiret, EBSCOhost, Scielo, Dialnet y Redalyc y la base de datos del Banco Mundial. En la búsqueda electrónica se usaron como descriptores los términos: trabajo infantil; educación y trabajo infantil; salud y trabajo infantil; mercados imperfectos y trabajo infantil; programas de transferencias $y$ trabajo infantil; migraciones y trabajo infantil, en inglés y español, ya sea que se encuentren en todo el texto, en el resumen y/o en el titulo. La selección de los descriptores se realizó con base a los resultados de una búsqueda sistemática previa usando como descriptor el término trabajo infantil, tema central del presente artículo. Dicha búsqueda previa permitió evidenciar los mayores desarrollos teóricos y empíricos del trabajo infantil, permitiendo en consecuencia, la selección de los descriptores finales. A su vez, la selección de los estudios se hizo con base a la relevancia del título y/o resumen.

El presente documento consta de siete secciones, incluida la presente introducción. En la segunda sección, se exponen los fundamentos teóricos que constituyen la base para el desarrollo empírico posterior. En la tercera, se muestran los principales trabajos que abordan la relación entre el trabajo de los niños y la educación, mientras que en la cuarta, se detallan los estudios que analizan la relación entre el trabajo y la salud. En la quinta sección, se analizan otras aproximaciones que incluyen el examen del papel de los mercados imperfectos y los programas de transferencias condicionadas sobre el trabajo infantil. Finalmente, se esbozan las conclusiones.

\section{Fundamentos teóricos}

El abordaje teórico de las decisiones a las que se enfrenta una familia ha estado enmarcado tradicionalmente en el enfoque de producción de los hogares de Mincer (1963) y Becker (1965), con el cual se analiza la asignación del tiempo de las familias bajo el supuesto de que estas son agentes tanto productoras como maximizadoras de utilidad. En el modelo inicial, las familias combinan tiempo $y$ bienes de mercado para producir bienes y servi- cios que afectan directamente su función de utilidad, entre estos, los hijos y bienes de consumo como la comida o el alojamiento. En la "producción" de los hijos, el principal insumo es el tiempo que supone el cuidado de estos.

La extensión y aplicación del modelo de producción de los hogares a las decisiones de escolaridad y trabajo de los niños se encuentra en Rosenzweig \& Evenson (1977), quienes desarrollaron un modelo en el que las familias toman simultáneamente las decisiones de fertilidad y la asignación del tiempo de los niños entre el trabajo y la escuela. Estas decisiones, según Rosenzweig \& Evenson (1977), dependen de las características del hogar y de las preferencias y las restricciones de recursos a las que se enfrentan los padres. La aplicación empírica de este modelo para la India muestra que la asignación del tiempo de los niños depende de los salario de mercado: entre más alto salario de la madre y más bajo el salario del niño se alcanza menor tasa de fertilidad, mayor educación del niño y menor trabajo infantil.

El reciente "boom" de la literatura puede remontarse al trabajo teórico de Basu \& Van (1998), quienes argumentan que para todos los hogares existe un salario critico el cual determina si el hogar envía los niños al mercado laboral. Concretamente, el hogar sólo enviará sus niños a trabajar si el salario masculino adulto está por debajo de ese salario crítico. Esta premisa, que constituye uno de los supuestos básicos del modelo de Basu \& Van (1998), es conocida como la hipótesis de "Luxury axiom", término que es usado actualmente para agrupar los trabajos empíricos que analizan la relación entre los ingresos o pobreza y el trabajo infantil. Aunque esta relación ha sido propuesta en términos teóricos y analíticos, los estudios empíricos la han encontrado muy difícil de sostener.

Una conclusión importante del estudio de Basu \& Van (1998) se origina del segundo supuesto del modelo: "Substitution axiom", el cual considera que el trabajo de los niños y de los adultos son sustituibles en la producción. Este supuesto permite al mercado laboral estar caracterizado por múltiples equilibrios: unos en los cuales los salarios son bajos y los niños 
trabajan, y otros en los cuales los salarios son altos y los niños no trabajan. En este sentido, el efecto de la prohibición total del trabajo infantil sería una reducción de este debido a que el salario de los adultos aumenta en respuesta al exceso de la demanda de trabajo y ante este aumento en los salarios es probable que los padres no quieran enviar a sus hijos a trabajar.

La aparición del trabajo de Baland \& Robinson (2000) refuta la idea de la relación positiva de los ingresos del hogar en el trabajo infantil. El modelo de equilibrio general construido por estos autores considera dos periodos y está cimentado bajo el concepto de altruismo tanto de los padres como de hijos. Específicamente, se considera que los adultos que crecieron un hogar pobre realizan transferencias a sus padres, quienes las reciben como una retribución al ingreso perdido y al tiempo destinado a la educación del niño en lugar del trabajo. Por tanto, ante un aumento en los ingresos del hogar se llega a un equilibrio en el que los niños no transfieren dinero a sus padres, y cuando esto pasa el trabajo infantil aumenta.

\section{Evidencia empírica de la hipótesis de "Luxury axiom"}

Desde la publicación del trabajo seminal de Basu \& Van (1998) ha surgido un importante cuerpo empírico que analiza el impacto de los ingresos de los padres o la pobreza del hogar sobre el trabajo de los niños. Usando variables monetarias, Kambhampati \& Rajan (2005) confirman esta hipótesis para la India: un incremento en el salario de padres y madres reduce el trabajo infantil. No obstante, el efecto no es ni continúo ni monótono para el caso del salario de la madre. En esta misma línea, Edmonds (2005) encuentra que los mejoramientos en el gasto per cápita en Vietnam pueden explicar el 80\% de la caída en el trabajo infantil en los hogares cuyo gasto mejora lo suficiente como para superar la pobreza. Para probar estar hipótesis Edmonds (2005) utilizó una descomposición no paramétrica con datos panel que abarca los periodos de crecimiento de Vietnam.
Tradicionalmente el discurso académico y político ha considerado a la pobreza como el principal factor que empuja a los hogares a enviar a sus niños al mercado laboral. Entre los organismos internacionales que apoyan esta idea se encuentran la Organización Internacional de Trabajo (OIT), El Fondo de las Naciones Unidas para la infancia (UNICEF), e incluso el Instituto de Formación para Educadores de Jóvenes, Adolescentes y Niños Trabajadores de América Latina y el Caribe (IFEJANT), institución que apoya y promueve el trabajo infantil por considerarlo, como cualquier otra actividad, parte de lo cotidiano y que favorece la consolidación de una imagen positiva (Rausky, 2009).

Esta hipótesis ha sido confirmada analíticamente por tratadistas como Admassie (2002), quien encuentra que la alta tasa de trabajo infantil en la región sub - Sahariana de África puede ser explicada, entre otras cosas, en términos de la alta incidencia de la pobreza. Amin, Quayes \& Rives (2004) al estudiar los determinantes del trabajo infantil en Bangladesh, apoyan la noción de que la pobreza de la familia afecta la probabilidad que un niño trabaje. Del Rio \& Cumsille (2008), a través de una regresión logística para Chile, encuentran que las razones económicas aún son el principal motor en algunos grupos más vulnerables, pero las preferencias culturales y la participación de los adolescentes en esta decisión está tomando mayor fuerza. Para Noceti (2009), el trabajo infantil en Bahía Blanca (Argentina) es un tipo de estrategia de supervivencia seleccionada por el grupo familiar.

Si bien la pobreza ha sido encontrada como uno de los determinantes más robustos del trabajo infantil, varios autores (Bhalotra \& Heady, 2001; Basu, Das \& Butta, 2007; Bar \& Basu, 2009) han llamado la atención de lo que ellos denominan "la paradoja de la riqueza". Esta hace referencia a que a medida que la tenencia de la tierra aumenta el trabajo infantil también lo hace mostrando una relación $U$ invertida, lo que pone en duda la hipótesis de la pobreza como el principal determinante del trabajo infantil. Ávila (2009) concilia esta cuestión al sugerir que el trabajo infantil obedece a un razonamiento distinto si corresponde a un trabajo fuera o dentro del hogar: en Colombia, para el trabajo fuera del hogar 
se satisface la hipótesis del luxury axiom (a menor riqueza mayor trabajo infantil); para el trabajo en un negocio familiar la relación es opuesta: a mayor riqueza, mayor trabajo de los menores. Por otra parte, Bhalotra \& Heady (2001) sostienen que la paradoja de la riqueza puede ser explicada por fallas en el mercado laboral y de la tierra.

Otro grupo de trabajos empíricos demuestra la invalidez del luxury axiom para explicar la persistencia del trabajo infantil. Dentro de estos se encuentra el de Ray (2000), cuyos resultados rechazan las hipótesis en Pakistán y sugieren que el ingreso y las variables relacionadas no tienen un efecto negativo esperado sobre la participación de los niños en el mercado laboral. Para Perú la relación es débil. Al usar las horas trabajadas, Ray observó una relación directa entre estas y la pobreza para Pakistán pero no para Perú.

Los resultados de Ray (2000), han sido respaldados por Emerson \& Souza (2003) quienes encuentran que mientras el ingreso tiene un efecto significativo sobre la transmisión del trabajo infantil, por sí solo no puede explicar el grado de persistencia. En esta misma línea, Emerson \& Knabb (2006) al llevar a cabo un examen de la persistencia intergeneracional del trabajo infantil encuentran que la pobreza por sí sola no puede ser la causa del trabajo infantil. Este estudio además presenta un modelo que propone un mecanismo diferente a través del cual el trabajo infantil puede ser transmitido a través de las generaciones de una familia: diferencias en las oportunidades. Si las diferencias en las oportunidades existen se muestra que las familias pueden llegar a estar atrapadas en el trabajo infantil.

Con un estudio de caso French \& Wokutch (2005) también rechazan la hipótesis "luxury axiom", al encontrar que las causas más importantes de los empleos de los niños trabajadores de la industria de calzado de la región de Franca en Brasil parecen tener menos que ver con la pobreza o la persistencia de las tradiciones y mucho más con la manera en la cual esta industria y sus trabajadores son integrados en el orden global emergente. Sin embargo, con una muestra de varios países Cigno, Rosati \& Guarcello (2002) no encuentran una relación entre la globalización (medida por los tratados internacionales) y el trabajo infantil.

Otra vía que ha sido sostenida en la literatura para refutar a la hipótesis, se encuentra en Rogers \& Swinnerton (2004), quienes bajo el marco del modelo de Baland \& Robinson (2000) y asumiendo altruismo tanto de los padres como de los hijos sostienen que incrementos en los ingresos de los padres no conduce a una disminución en el trabajo infantil. Bhalotra (2002) intenta conocer el grado de altruismo de los padres en Pakistán.

Los resultados a veces opuestos entre los estudios que analizan la hipótesis "luxury axiom" obedecen a que, según Kambhampati \& Rajan (2005), el ingreso sólo es uno de los tantos determinantes del trabajo infantil, además, la manera en la cual se mide el ingreso también puede afectar los resultados: ingresos salariales, ingresos no-salariales, línea de pobreza, GDP o los gastos. Por otra parte, el ingreso del hogar puede ser una variable endógena porque puede estar determinado por el trabajo del niño.

\section{Trabajo Infantil y educación}

A menudo se considera que la consecuencia de mayor trascendencia del trabajo infantil es el sacrificio del capital humano cuyos efectos se manifiestan en mayor medida en el bienestar del niño a largo plazo, ya que como lo sugiere Bacolod \& Ranjan (2008): un niño que adquiere menos educación debido a su trabajo crece para ser pobre de adulto y como adulto pobre enviará a sus niños al mercado laboral, perpetuando el ciclo entre pobreza. El trabajo infantil, además, repercute en el desarrollo del país ya que la productividad futura de este depende principalmente de la inversión en capital humano actual.

El marco del Programa Internacional para la Erradicación del Trabajo Infantil - IPEC de la Oficina Internacional del Trabajo - OIT (ILO-IPEC), la educación es vista como el principal factor para reducir o eliminar el trabajo infantil, en especial, la educación primaria. Es así como la ILO-IPEC, ha adelantado proyectos para estimar los beneficios y costos de impartir una educación primaria universal y una 
mayor educación secundaria, con lo que se esperaría la reducción del trabajo infantil en el mundo. En particular, Matz (2002) estima que los beneficios de emprender esta tarea podrían ser tres veces mayores a los costos, incluso bajo el reconocimiento de que los beneficios podrían estar subestimados.

En la literatura existe un creciente cuerpo empírico que trata de analizar la naturaleza de la relación negativa entre la escolaridad y trabajo infantil, actividades excluyentes debido a que compiten por el tiempo del niño. En especial, los estudios en este sentido se han concentrado en la relación entre el trabajo infantil y las siguientes variables relacionadas con la educación: asistencia a la escuela, rendimiento académico y horas dedicadas al estudio.

Dentro del primer grupo de trabajos se destaca el de Canagarajah \& Coulombe (1997) quienes encuentran evidencia de una relación negativa y significativa entre la asistencia a la escuela e ir a trabajar en Ghana. Khanam (2004); Khanam (2007); y Khanam \& Ross (2008) encuentran que el trabajo infantil afecta negativa a la educación del niño, lo cual se refleja en una menor asistencia a la escuela en Bangladesh y un menor rendimiento escolar. Fallon \& Tzannatos (1998) plantean que la correlación negativa entre la asistencia escolar y trabajo infantil se evidencia en los países de bajos ingresos, pero en los países con más de US\$600 la correlación negativa cesa, a pesar de la alta variabilidad de los datos.

En cuanto a los trabajos empíricos que analizan las repercusiones del trabajo infantil sobre el rendimiento académico de los menores se encuentra el de Psacharopoulos (1997) quien argumenta que un niño que trabaja reduce su rendimiento académico por cerca de 2 años de educación comparado con el grupo de control (niños que no trabajan). Asimismo, la repetición de cursos, un fenómeno común en Latinoamérica, está cercanamente asociada al trabajo infantil. Akabayashi \& Psacharopoulos (1999) señalan que la ocupación de los niños esta negativamente relacionadas con las destrezas en lectura y matemáticas. Cervini (2005), encuentra evidencia de que el rendimiento promedio en matemáticas de los alumnos de séptimo año en el área urbana en Argentina es un 20\% menor para los niños que trabajan fuera de casa y por cuatro o más horas en contraposición con los no trabajan. Para Binder\& Scrogin (1999) las horas de trabajo no afectan al rendimiento escolar en México.

Aunque la mayor parte de la literatura se ha centrado sobre los determinantes de las decisiones de los hogares sobre si enviar los niños al trabajo o a la escuela (Rosati \& Rossi, 2003), en los últimos años ha crecido el interés sobre la cantidad de tiempo que los niños dedican al trabajo y/o a la educación. Al respecto, Akabayashi \& Psacharopoulos (1999) encuentran que existe trade off entre las horas de trabajo y las horas de estudio en Tanzania, aunque las primeras dependen más de las condiciones socioeconómicas del niño que de las horas de estudio. Boozer \& Suri (2001) con un modelo panel basado en la aproximación de Deaton (1985), encuentran que una hora de trabajo reducen las horas de asistencia escolar en 0.38 horas. Usando el método de mínimos cuadrados estiman que una hora de trabajo reduce las horas de asistencia escolar en 0.21 horas. Mientras que Binder \& Scrogin (1999) encuentra que las horas dedicadas a laborar al parecer tiene un efecto pequeño en las horas destinadas a acumular capital humano.

Por otro lado, Lancaster \& Ray (2005) examinan el efecto de las horas de trabajo tanto en la tasa de matricula escolar como en las horas dedicadas al estudio en siete países ${ }^{1}$. La conclusión principal es que el trabajo infantil, aunque dure pocas horas, va en detrimento de ambas variables. Sin embargo, el efecto el impacto marginal del trabajo infantil es más perjudicial para las niñas que para los niños. Por el contrario, French (2010) no encontró evidencia entre el rendimiento y la asistencia a la escuela y el trabajo infantil para los trabajadores de una fábrica de zapatos en Brasil.

Algunos trabajos han mostrado que la relación entre las horas trabajadas y la escolaridad no presentan una relación lineal. Admassie \& Bedi (2003) encon-

1 Belice, Camboya, Filipinas, Namibia, Panamá, Portugal y Sri Lanka. 
tró una relación no lineal entre las horas de trabajo y asistencia escolar/rendimiento en lectura y escritura de los niños del área rural de Etiopia: inicialmente existe una relación positiva entre el trabajo y la asistencia/ rendimiento, sin embargo, entre $16-$ 22 horas de trabajo semanal, la habilidad para leer y escribir comienza a sufrir mientras que la asistencia no se afecta. Más allá de ese límite, la asistencia y la habilidad en lectura y escritura comienzan a sufrir. Rosati \& Rossi (2003) argumentan que la cantidad de horas trabajadas son un importante determinante del rendimiento escolar en Pakistán y Nicaragua: pocas horas de trabajo incrementa la probabilidad de retrasarse en el curso en un 10\%, aunque las primeras horas de trabajo tienen un mayor impacto en el rendimiento que las sucesivas, lo que muestra que la relación no es monótona creciente.

Otros enfoques bajo la línea de pensamiento de Myers (2001) sugieren que el trabajo infantil puede constituir un componente importante en la crianza de los niños ya que motiva la construcción de valores que les serán de gran utilidad en la adultez para su desenvolvimiento laboral, entre ellos, la responsabilidad, la autonomía y la perseverancia. Si bien estos enfoques reconocen que algunas formas de trabajo infantil son producto de las distorsiones económicas existentes en un país en vías de desarrollo, sus tratadistas plantean que la adquisición de habilidades, aptitudes y capacidades a temprana edad compensan las pérdidas estimadas por la no formación del capital humano.

Independiente de la naturaleza de la relación entre la educación y el trabajo infantil - directa o indirecta, causal o espuria - la literatura destaca los altos costos de la educación y la baja calidad como uno de los principales determinantes de las decisiones del hogar sobre si enviar los niños a la escuela o al mercado laboral (Jensen \& Nielsen (1997); Canagarajah \& Coulombe (1997). Además, la poca o nula oferta de escuelas (Khanam \& Ross, 2008) y la débil relevancia de la educación (Canagarajah \& Coulombe (1997) han presionado a muchos niños al mercado de trabajo.

Las características de la familia tienen un importante papel en la decisión de estudiar o trabajar, especial- mente la educación de los padres (Canagarajah \& Coulombe, 1997; Jensen \& Nielsen, 1997; Khanam, 2004; Kambhampati \& Rajan, 2005); Khanam, 2007; Khanam \& Ross, 2008) y el estatus laboral de los miembros del hogar. Con respecto a este último, Bernal \& Cárdenas (2006) encuentran que para Colombia entre mayor tasa de ocupación de los adultos del hogar, mayor es la probabilidad que un niño trabaje. Este resultado es corroborado por Kambhampati \& Rajan (2005) para el caso de las niñas en India y por Urueña, Tovar \& Castillo (2009) para el departamento del Valle del Cauca (Colombia).

Finalmente, para formular políticas que mitiguen o eliminen la problemática del trabajo infantil, deben tomarse en cuenta las diversidades regionales y las peculiaridades de la zona de estudio. Por ejemplo, mientras que la provisión de calidad de escuela ayuda a reducir el trabajo infantil en todos los lugares, tales escuelas necesitan ser ubicados cerca del empleo de los niños en Perú y cerca del barrio de residencia en Pakistán (Maitra \& Ray, 2002).

\section{Trabajo infantil y salud}

Otra de las cuestiones que más ha llamado la atención de los investigadores del trabajo infantil es el impacto que el trabajo tiene sobre la salud de los menores. La literatura en este sentido plantea que los niños son mucho más vulnerables que los adultos a los factores de riesgo debido a que se encuentran en etapa de desarrollo físico y psicológico, además de que son más propensos a ser explotados en largas jornadas laborales con salarios bajos.

En esta dirección, Satyanararayanan, Krishna \& Rao (1986) encuentran que los niños que trabajan presentan déficit en el crecimiento en relación con los que asisten a la escuela para el área rural de India; French (2010) empleando un estudio de caso en Brasil sugiere que el trabajo en la fábrica de zapatos afecta la salud de los niños. Si bien Immink \& Payongayong (1999) encontraron que en Guatemala la participación de los niños en edad escolar en las fincas no está asociada con una reducción en su propio crecimiento y desarrollo, los hermanos más 
jóvenes si experimentaron déficit en el crecimiento, mostrando que el trabajo infantil puede generar un efecto indirecto negativo sobre la salud de los hermanos operando a través de la relocalización de los recursos.

Para el caso de los riesgos asociados a los trabajos en las plazas de mercado, Vargas \& Restrepo (2002) realizan una evaluación ergonómica validada en Colombia, la cual demuestra que todos los oficios infantiles o juveniles presentan altas cargas físicas psicosociales. Estos oficios, por lo general, requieren un importante esfuerzo físico para la carga pesada de transporte, embalaje y ubicación de los productos que comercian. Estos resultados fueron confirmados por Briceño \& Pinzón (2005) para la plaza de mercado de Bogotá (Colombia); y Rojas, Vegas, Briceño \& Rodríguez (2010) para el mercado público de Valencia (Venezuela).

Contrario a la presunción general y a los hallazgos de los estudios empíricos sobre los efectos adversos del trabajo sobre la salud de los niños, la literatura también ha documentado efectos positivos del trabajo infantil sobre la salud. Hincapié (2007), por ejemplo, encuentra que el trabajo infanto-juvenil tiene un efecto positivo sobre la nutrición de los niños colombianos entre 6 y 17 años, a través de la generación directa de ingreso extra para el hogar, o al permitir que se libere mano de obra adulta de los trabajos del hogar. Para O'Donnel, Rosati \& VanDoorslaer (2002) este es un argumento persuasivo y plausible sólo en condiciones de extrema pobreza.

Las consecuencias del trabajo infantil no sólo son inmediatas, sino que pueden presentarse varios años después de realizada la actividad. Al respecto, Rosati \& Straub (2007) argumentan que muchos de los riesgos de la salud del trabajo infantil son más probables a ser manifestados en la adultez principalmente aquellos originados por la exposición a pesticidas, químicos, polvos y agentes cancerígenos que incrementan el riesgo de desarrollar problemas bronquiales, canceres y una variedad de enfermedades.

Entre los estudios que abordan las consecuencias de largo plazo del trabajo infantil sobre la salud se destacan los de O'Donnel, Rosati, \& vanDoorslaer (2005) para Vietnam, quienes encuentran que algunas labores pueden aumentar el riesgo de enfermedad en los 5 años siguientes al trabajo; Kassouf, McKee \& Mossialos (2001) sugieren que entre más joven una persona comienza a trabajar en Brasil más alta la probabilidad de reportar mala salud cuando se es adulto. Si se controla por la educación o el ingreso del hogar se reduce sustancialmente el efecto pero no se elimina. Este resultado también es consistente con los hallazgos de Rosati \& Straub (2007) para Guatemala, quienes argumentan que la edad a la un niño comienza a trabajar tiene un efecto sobre la salud del adulto.

Si bien la mayoría de los trabajos encuentra un impacto - negativo o positivo, de corto o largo plazo del trabajo de los menores sobre la salud, es posible encontrar en la literatura un grupo de análisis empíricos que sugieren que la relación es nula o espuria, entre estos, Beegle, Dehejia \& Gatti (2004) quienes encuentran que no parece haber un efecto de la participación en el mercado laboral de los niños en la salud en Vietnam; Fentiman, Hall \& Bundy (2001) no encuentran diferencias en el crecimiento entre los niños que están matriculados y los que no en el área rural de Ghana; Cigno \& Rosati (2001) no encuentra evidencia de que el trabajo infantil afecte a la salud para el área rural de la India.

\section{Otras aproximaciones}

\subsection{El papel de los mercados de capitales imperfectos}

En los últimos años, la literatura ha destacado el papel de otros factores en la explicación del trabajo infantil, cuyo análisis tiene importantes implicaciones de política ya que permite en algunos casos mitigar el problema con alcances de más a corto plazo. Uno de estos factores, poco estudiado pero con creciente importancia, lo constituye las imperfecciones en el mercado de capitales.

De acuerdo con la evidencia empírica y los desarrollos teóricos, el mercado de capitales es un sustituto del trabajo infantil ya que cuando las familias en- 
frentan choques económicos y no pueden acceder al mercado crediticio, los niños se convierten en un activo para suavizar el consumo (López, 2001). En otras palabras, las imperfecciones en el mercado financiero, al interferir en la capacidad de los padres para hacer intercambios entre ingreso actual y futuro, presionan hacia un mayor trabajo infantil.

Esta hipótesis es sostenida por Jensen \& Nielsen (1997) quienes argumentan que la falta de acceso al crédito induce a los padres a no enviar a sus niños a la escuela. Dehejia \& Gatti (2002) tomando una muestra de varios países, encuentran que hay una relación negativa significativa entre el trabajo infantil y las restricciones en el crédito (medidas por el desarrollo financiero del país). Esta relación es particularmente fuerte en los países de bajos ingresos. Beegle, Dehejia \& Gatti (2003) encuentran que los choques o variaciones transitorios en el ingreso (medida por las pérdidas accidentales en las cosechas) conducen a incrementos en el trabajo infantil en Tanzania y que el acceso al crédito (medido por la tenencia de activos pignorables) mitiga este efecto.

Por otro lado, Guarcello, Mealli \& Rosati (2002) encuentran que los efectos más importantes de las restricciones crediticias se presentan principalmente en la decisión de que los niños no realicen alguna actividad (estudiar o trabajar) o enviarlos a la escuela. Sin embargo, las restricciones pueden afectar indirectamente al trabajo infantil ya que los niños que no realizan alguna actividad tienen particularmente el riesgo de convertirse en trabajadores. Es de destacar que los mejoramientos en el acceso al crédito no sólo reducirían la oferta de trabajo infantil, sino que al constituir el crédito un importante determinante en la inversión de las firmas, reducirían la demanda laboral de los niños (Dehejia \& Gatti, 2005).

\subsection{Migración y trabajo infantil}

Si bien la relación entre migración (por desplazamiento interno o movilidad internacional) y trabajo infantil ha sido poco abordado por la literatura empírica, su importancia se hace mas latente con fenómenos como la globalización económica y la consecuente desaparición de las fronteras. En este escenario, la naturaleza y gravedad del trabajo in- fantil dependen del tipo de migraciones que se considere, en especial, aquellas migraciones en las que los niños viajan autónomamente, suelen ser las más perjudiciales (Casteras, 2009).

El trabajo infantil es en ocasiones visto como una estrategia de inserción y adaptación de la familia a su nuevo entorno, y se convierte en un medio indispensable para la subsistencia de esta. Esta idea es apoyada por estudios analíticos como los de Monroy y Juan, 2009 para el caso de los migrantes de la comunidad indígena de Coatecas (México); por Pinzón et al. (2003) para los trabajadores ambulantes en las calles de Bogotá; y por Pinzón et al. (2006) para los niños trabajadores ambulantes en las ciudades capitales de América latina. Estos últimos estudios también ponen de relieve la magnitud del problema: cerca del 25 y 50\% de los niños trabajadores ambulantes en las ciudades capitales de América latina manifiestan ser desplazados por la violencia o por miseria (Pinzón et al., 2006), mientras que para los trabajadores ambulantes en las calles de Bogotá este porcentaje se sitúa en 50\% (Pinzón et al., 2003).

\subsection{Eficiencia de los programas de transferencias}

Otra parte de la literatura estudia la eficacia de los programas de transferencia de efectivo en países de bajos ingresos sobre la educación y el trabajo infantil. Al respecto, Schultz (2004), y Skoufias \& Parker (2001), encuentran que el programa de transferencias condicionadas en México muestra efectos significativos sobre ambas actividades. Edmonds (2005), por su parte, encuentra que la recepción de grandes transferencias de efectivos incrementó significativamente la educación y redujeron el trabajo infantil en Sur África, pero la reducción en el trabajo infantil fue pequeña. Bourguignon, Ferreira \& Leite (2003) encontraron los mismos resultados para Brasil. Bernal \& Cárdenas (2006) para el caso de Colombia evalúan el impacto del programa de subsidios condicionados de "familias en acción" sobre el trabajo infantil, encontrando que este ha sido una estrategia efectiva para la reducción del problema, particularmente en el caso de los niños varones. 
No obstante, Borraz \& González (2009), encuentran evidencia de que el programa de transferencia monetaria condicionada implementado en Uruguay no ha tenido efectos en la asistencia escolar ni en el trabajo infantil.

\section{Consideraciones Finales}

Del ejercicio de la revisión sistemática de la literatura del trabajo infantil se extraen conclusiones y consideraciones importantes. En primer lugar, se destaca que la literatura ha estado dirigida principalmente al estudio de la relación entre el trabajo de los menores y la educación y la salud. Estas relaciones exhiben una naturaleza compleja debido a que no existe una contundente evidencia de los efectos - negativos o positivos, de largo o corto plazo, causales o espurios - del trabajo de los menores sobre estas variables. Todo esto hace que el análisis empírico sea un ejercicio difícil.

Por otro lado, aunque la pobreza sea tratada como el principal determinante del trabajo infantil, los desarrollos más recientes de la literatura han puesto en duda esta hipótesis, al encontrar una relación de la forma $U$ invertida de la tenencia de la tierra y el trabajo infantil. Además, han surgido otros factores que explican este fenómeno y que podrían tener importantes implicaciones de política pública, entre estos, los mercados imperfectos, las migraciones y los programas de transferencias.

De la revisión también se destaca que la mayor parte de los trabajos se ha enfocado en los determinantes por el lado de la oferta de trabajo infantil, es decir, por aquellos factores que presionan a los hogares a enviar a sus niños al trabajo, en lugar de los determinantes por el lado de la demanda, es decir, los factores que hacen que las firmas empleen mano de obra infantil.

\section{Referencias}

Admassie, A. (2002). Explaining the High Incidence of Child Labour in Sub-Saharan Africa". En: African Development Review, 14 (2): 251-275.
Admassie, A \& Bedi, A.S. (2003). Attending School, Two "Rs" and Child Work in Rural Ethiopia". En: ISS Working Paper, General Series, No. 387.

Akabayashi, H \& Psacharopoulos, G. (1999). The Trade-off between Child Labour and Human Capital Formation: A Tanzanian Case Study". En: Journal of Development Studies, 35 (5): 120-140.

Amin, S, Quayes, M. \& Rives, J. (2004). Poverty and Other Determinants of Child Labor in Bangladesh". En: Southern Economic Journal, 70 (4): 876-892.

Bacolod, M. \& Ranjan, P. (2008). Why children Work, Attend School, or Stay Idle: The Roles of Ability and Household Wealth". En: Economic Development \& Cultural Change, 56(4):791-828.

Baland, J \& Robinson, J. (2000). Is Child Labor Inefficient? En: Journal of Political Economy, 108(4):663.

Bar, T. \& Basu, K. (2009). Children, Education, Labor, And Land: In The Long Run And Short Run .En: Journal of the European Economic Association, 7 (2/3):487-497.

Basu, K. \& Hoang Van, P. (1998). The Economics of Child Labor. En: American Economic Review, 89 (5):1386-1388.

Basu, K. (1999). Child Labor: Cause, Consequence, and Cure, with Remarks on International Labor Standards. En: Journal of Economic Literature, 37 (3):1083-1119.

Basu, K, Das, S. \& Dutta, B. (2007). Child Labor and Household Wealth: Theory and Empirical Evidence of an Inverted-U. En: IZA. Discussion Paper No. 2736.

Becker, G. (1965). A theory of the allocation of time. En: Economic Journal, 75 (299): 493-517.

Beegle, K, Dehejia, R. \& Gatti, R. (2003).Child Labor, Crop Shocks, and Credit Constraints. En: National Bureau of Economic Research. Working Paper Series № 10088. Disponible en http://www.nber.org/papers/w10088.

Beegle, K, Dehejia, R \& Gatti, R. (2004). Why Should We Care About Child Labor? The Education, Labor Market, and Health Consequences of Child Labor. En: NBER Working Papers 10980, National Bureau of Economic Research, Inc.: 1-52.

Bernal, R. \& Cárdenas, M. (2006). Trabajo infantil en Colombia. Fedesarrollo. Disponible en http://www.fedesarrollo.org. co/includes/scripts/open.asp?ruta=/images/dynamic/articles/521/Cardenas.pdf

Bhalotra, S. (2002). Parent Altruism. Royal Economic Society Annual Conference 2002 25, Royal Economic Society.

Bhalotra, S. \& Heady, C. (2001). Child farm labour: the wealth paradox. En:Social Protection Discussion Papers 24088, The World Bank

Binder, M. \& Scrogin, D. (1999). Labor force participation and household work of urban schoolchildren in Mexico: Characteristics. En: Economic Development \& Cultural Change, 48(1):123

Boozer, M. \& Suri, T. (2001). Child Labor and Schooling Decisions in Ghana, Working Paper Yale University.

Borraz, F. \& González, N. (2009). Impact of the Uruguayan conditional cash transfer program .En: Cuaderno de economía 2009,46(134): 243-271.

Bourguignon, F, Ferreira F. \& Leite, P. (2003).Conditional Cash Transfers, Schooling y Child Labor: Micro-Simulating Brazil's BolsaEscola Program. En: World Bank Economic Review, 17 (2):229-54 
Briceño, L. \& Pinzón, Á. (2005). Trabajo infantil en una plaza de mercado de Bogotá, Colombia. En: Revista de Salud Pública, 7 (1): 26-38.

Canagarajah, S. \& Coulombe, H. (1997). Child Labour and Schooling in Ghana. En: Policy Research Working Paper No. 1844, World Bank, Washington, D.C.

Casteras, D. (2009). Efectos de la migración sobre el trabajo infantil en Colombia. Revista economía institucional, 11 (20): 229-252.

Cervini, R. (2005). Trabajo infantil urbano y logro en matemáticas de la educación básica. Un modelo de dos niveles. En: Revista mexicana de investigación educativa, 10(25): 451-480.

Cigno, A. \& Rosati, F.C. (2001).Child labour, education, fertility and survival in rural India. Pacific Economic Review, 7: 1-19.

Cigno, A, Rosati, F. \& Guarcello, L. (2002). Does Globalisation Increase Child Labour? . En:IZA Discussion Papers 470, Institute for the Study of Labor (IZA).

Dehejia, R. \& Gatti, R. (2002). Child Labor: The Role of Income Variability and Access to Credit Across Countries. En: World Bank Policy Research Working Paper no.2767 and National Bureau of Economic Research Working Paper No. 9018.

Dehejia, R. \& Gatti, R. (2005). Child Labor: The Role of Financial Development and Income Variability across Countries. En: Economic Development and Cultural Change, University of Chicago Press, 53(4): 913-32.

Del Rio, M. \& Cumsille, P. (2008). ¿Necesidad Económica o Preferencias Culturales? La Justificación Parental del Trabajo Infantil en Chile. En: Psykhe, 17(2): 41-52.

Emerson, P. \& Souza, A. (2003). Is There a Child Labor Trap? Intergeneratio.nal Persistence of Child Labor in Brazil .En: Economic Development \& Cultural Change, 51(2): 375.

Emerson, P. \& Knabb, S. (2006). Opportunity, Inequality and the Intergenerational Transmission of Child Labour .En: Economica, 73 (291):413-434.

Edmonds, E. (2005). Does Child Labor Decline with Improving Economic Status. En: Journal of Human Resources, 40 (1):77-99.

Fallon, P. \& Tzannatos, Z. (1998). Child Labor: Issues and Directions for the World Bank. Human Development Network. Washington: World Bank.

Fentiman, A, Hall, A. \& Bundy, D. (2001). Health and cultural factors associated with enrolment in basic education: A study in rural Ghana. En: Social Science and Medicine, 52:429- 439.

French, J. \& Wokutch, R. (2005). Child Workers, Globalization, and International Business Ethics: A Case Study In Brazil's Export-Oriented Shoe Industry .En: Business Ethics Quarterly, 15(4):615-640.

French, J. (2009).Children's Labor Market Involvement, Household Work, and Welfare: A Brazilian Case Study .En: Journal of Business Ethics, 92 (1):63-78.

Guarcello, L, Mealli, F. \& Rosati, F.C. (2002).Household Vulnerability and Child Labor: The Effect of Shocks, Credit Rationing and Insurance .Manuscript, InnocentiResearch Center, UNICEF.

Hincapié, D. (2007).El trabajo infanto-juvenil y el estado nutricional de los menores colombianos. En: Desarrollo y Sociedad, 59: $63-115$

Immink, M. \& Payongayong, E. (1999).Risk analysis of poor health and growth failure of children in the central highlands of Guatemala En: Social Science \& Medicine, Elsevier, 48(8): 997-1009.

Jensen, P. \& Nielsen, H. S. (1997). Child Labour or School Attendance?Evidence from Zambia .En: Journal of Population Economics, 10: 407-424.

Kambhampati, U. \& Rajan, R. (2005). Does Child Work Decrease with Parental Income? The Luxury Axiom Revisited in India. En: European Journal of Development Research, 17 (4): 649-680.

Khanam, R. (2004). Impact of Child Labour on School Attendance and School Attainment: Evidence from Bangladesh. 3rd International Conference of the Japan Economic Policy Association (JEPA), Meiji University, Tokyo, Japan, Nov. 13-14.

Khanam, R. (2007).Child Labour and School Attendance: Evidence from Bangladesh. En: International Journal Social Economics, 35(1): 77-98

Khanam, R. \& Ross, R. (2008). Child Work and Other Determinants of School Attendance and School Attainment in Bangladesh. Proceedings of the 37th Australian Conference of Economists, Goldcoast, Australia. Paper No. 9397.

Kassouf, A. L, McKee, M. \& Mossialos, E. (2001). Early entrance to the job market and its effects on adult health: Evidence from Brazil, health policy and planning. En: HealthPolicy and Planning, 16 (1): 21-28.

Lancaster, G. \& Ray, Ranjan (2005). Efectos del trabajo infantil en la escolaridad: estudio plurinacional. En: Revista internacional del trabajo, 124(2):209-232.

López Calva, L. (2001). Child Labor: Myths, Theories and Facts. En: Journal of International Affairs, 55 (1): 59.

López, D. (2009). Pobreza y trabajo infantil: diferencias entre trabajo dentro y fuera del hogar. Evidencia para Colombia, 2001 - 2003. En: Documentos de Economía, 2009: 1-32.

Maitra, P. \& Ray, R. (2002). The Joint Estimation of Child Participation in Schooling and Employment: Comparative Evidence from Three Continents. En: Oxford Development Studies, 30(1): 41-62.

Matz, P. (2002). Costs and Benefits of Education to Replace Child Labour. IPEC-ILO.Working Paper.

Myers, W.E. (2001). The right rights? Child labour in a globalising world. En: Annals of the American Academy of Political and Social Science, 575: 38-55.

Mincer, J. (1963). Market Prices, Opportunity Cost and Income Effects .Measurement in Economics. Carl Christ. Stanford, Stanford University Press.

Monroy, F. \& Juan, J. (2009). Agricultura y migración campesina: un estudio para comprender la incorporación del trabajo infantil en una región indígena de México. En: Revista argentina de sociología, 7(13): 125-149.

Noceti, M. (2009). El trabajo infantil como estrategia de sostén de las familias pobres en la Argentina, la necesidad de rediseñar el objeto de las políticas públicas. En:Acciones e investigaciones sociales, 27: 171-194.

O'Donnell, O, Rosati, F, \& van Doorslaer, E. (2002). Child labor and health: Evidence and research issues. Understanding Children's Work Discussion Paper.

O'Donnell, O, Rosati, F, \& van Doorslaer, E. (2005). Health effects of child work: Evidence from rural Vietnam .En:Journal of PopulationEconomics, Springer, 18 (3):437-467. 
Pinzón, A, Briceño, L, Gómez, A \& Latorre, C. (2003). Trabajo infantil en las calles de Bogotá. En: Rev. Cienc. Salud, 1(2): 151-163.

Pinzón, A, Briceño, L, Botero, J, Cabrera, P. \& Rodríguez, M. (2006). Trabajo infantil ambulante en las capitales latinoamericanas .En: Salud pública Méx, 48 (5): 363-372.

Psacharopoulos, G. (1997). Child labor versus educational attainment Some evidence from Latin America. En: Journal of Populationeconomics, 10 (4):377-386.

Rausky, M. (2009). ¿Infancia sin trabajo o Infancia trabajadora? Perspectivas sobre el trabajo infantil. En: Revista Latinoamericana de Ciencias Sociales, Niñez y Juventud, 7(2): 681-706.

Ray, R. (2000a). Analysis of child labour in Peru and Pakistan: A comparative study .En: Journal of Population Economics, $13(1): 3$.

Ray, R. (2000b). Poverty, household size and child welfare in India .En: Economic and Political Weekly (Bombay), 35(39): 3511-3520

Rogers, C. \& Swinnerton, K. (2004).Does Child Labor Decrease When Parental Incomes Rise? En: Journal of Political Economy, 112 (4): 939-946.

Rojas, M, Vegas, Z, Briceño, L. \& Rodríguez, L. (2010). Trabajo infantil y salud en un mercado público de Valencia, Venezuela. En: Revista de Salud Pública, 12 (1): 135-143.
Rosati, F. C. \& Rossi, M. (2003).Children's Working Hours and School Enrollment: Evidence from Pakistan and Nicaragua. En: The World Bank Economic Review, 17: 283-295.

Rosenzweig, M.\& Evenson, R. (1977).Fertility, Schooling and the Economic Contribution of Children in Rural India: An Econometric Analysis. En: Econometrica, 45 (5):1065-1079.

Satyanarayana, K, Krishna, T.P. \& Rao, B.S. (1986). The effect of early childhood undernutrition and child labour on the growth and adult nutritional status of rural Indian boys around Hyderabad. Human Nutrition and Clinical Nutrition 40C: 131-139.

Schultz, T. (2004). School Subsidies for the Poor: Evaluating the Mexican Progresa Poverty Program. En: Journal of Development Economics, 74 (1): 199-250.

Skoufias, E. \& Parker, S. (2001). Conditional Cash Transfers and Their Impact on Child Work and Schooling: Evidence from the Progresa Program in Mexico. En: Economía, 2 (1):45-96.

Urueña, S, Tovar, L. \& Castillo, M. (2009).Determinantes del trabajo infantil y la escolaridad: el caso del Valle del Cauca en Colombia. En: Revista latinoamericana de Ciencias sociales, Niñez y Juventud 7 (2): 707-733.

Vargas, A. \& Restrepo, H. (2002). Trabajo infantil y juvenil en una plaza de mercados de Medellín, año 2000. En: Rev Cubana Salud Pública, 28 (3) 